\title{
Field sensing using the magnetoresistance of IrMn exchange-biased tunnel junctions
}

\author{
D. Lacour ${ }^{\text {a) }}$, H. Jaffrès, F. Nguyen Van Dau, F. Petroff, A. Vaurès, and J. Humbert \\ Unité Mixte de Physique CNRS/THALES, Domaine de Corbeville, 91404 Orsay Cedex, \\ and Université Paris-Sud, 91405 Orsay Cedex, France
}

(Received 24 July 2001; accepted for publication 18 December 2001)

\begin{abstract}
An original concept of high sensitivity magnetic field sensor using the spin-dependent tunneling effect has been investigated. The required crossed-biased configuration is obtained by combining both shape energy originating from vicinal step bunched Si substrates and unidirectional exchange anisotropy supplied by an $\mathrm{Ir}_{20} \mathrm{Mn}_{80}$ film in the "top-biased" geometry. We demonstrate a linear and reversible signal at room temperature and above. The smooth loss of sensitivity at higher temperature is shown to be correlated to the thermal dependence of the exchange bias property when IrMn is deposited above the insulating $\mathrm{Al}_{2} \mathrm{O}_{3}$ barrier. (C) 2002 American Institute of Physics. [DOI: $10.1063 / 1.1450050$ ]
\end{abstract}

Since the work reported by Moodera et al. ${ }^{1}$ on the evidence of tunnel magnetoresistance at room temperature, the intensive research on the spin-dependent tunneling of magnetic junctions paves the way for a technology of integrated spin-electronics components such as magnetic memories $(\mathrm{MRAM})^{2}$ or various types of analog high-sensitivity field sensors (read heads, microcompass). ${ }^{3}$ Beyond the technological difficulties of synthetizing a very thin insulating film separating the two magnetic electrodes, what is required is to optimize the magnetic properties of each electrode. A parallel arrangement of both easy magnetization axes can lead to two different states of resistance perfectly adapted to the binary logic required by a MRAM-type functionality. To generate a highly linear and reversible analogical signal, required for field sensing, a crossed magnetization pattern has to be stabilized. ${ }^{4,5}$ Ideally, to realize a sensor, one has to prepare a very "soft" electrode, uniformly magnetized, behaving like a macrospin interacting with the applied field $H_{\text {ap }}$ to be detected. Moreover, the "hard" electrode, playing the role of spin analyzer, has to be locked along a perpendicular axis which defines the field sensitive direction. The only magnetic sensor based on spin-dependent tunneling under development uses a biasing field generated by a specific circuitry which is high power consuming. ${ }^{6,7}$ A way to circumvent this problem is to stabilize the specific noncollinear arrangement by using independent anisotropies induced within each electrode acting like an "internal" magnetic field (Fig. 1). This allows us to suppress the need of this specific circuitry and thus considerably reduces the power consumption.

In this letter, we discuss an original concept of highsensitive field sensor, which combines step anisotropy for the bottom electrode and unidirectional exchange bias anisotropy for the top electrode oriented perpendicularly to the step anisotropy. This provides the required perpendicular configuration. In details, the original method we propose consists of controlling the rotation of the "soft" Co electrode in its co-

a) Author to whom correspondence should be addressed; electronic mail: daniel.lacour@thalesgroup.com herent rotation regime and thus ensures the reversibility of the signal. This is made possible by taking advantage of the shape energy introduced by growing this electrode on a vicinal step-bunched $\mathrm{Si}(111)$ substrate misoriented by $4^{\circ}$ toward the $(11 \overline{2})$ direction, generating a uniaxial anisotropy $H_{K}^{\text {step }}$ along the larger axis of the steps. ${ }^{8}$ In this approach, the "hard" Co counterelectrode is maintained along the direction perpendicular to the steps by the unidirectional exchange bias effect. This is performed by depositing a $15 \mathrm{~nm}$ thick $\mathrm{Ir}_{20} \mathrm{Mn}_{80}$ layer on the "top" Co electrode. The exchange bias direction is adjusted by a thermal process under a field applied along the desired direction (the pinning direction). The direction of the exchange bias, controlled by the antiferromagnetic (AF) spins at the ferromagnetic $\mathrm{AF}$ interface, is determined by the field orientation during the annealing procedure in the vicinity the Néel temperature $T_{N}$ of the AF film. The $\operatorname{Ir}_{20} \mathrm{Mn}_{80}$ compound has been chosen because of its metallic character and its large transition temperature ${ }^{9}$ $\left(T_{N}^{\text {bulk }} \simeq 690 \mathrm{~K}\right)$. Note however that the principle of detection we have used (based on a softer anisotropy originating from the substrate) imposes a so-called "top" exchange-biased junction wherein the biased layer is the one deposited above the insulating barrier. The efficiency of this "top-biased" geometry (refer to Fig. 1) has not been addressed in the literature in the case of tunnel junctions devoted to sensing performance.

The whole stack $\left(\mathrm{Si}(111)_{4^{\circ}} / \mathrm{Co}(15 \mathrm{~nm}) / \mathrm{Al}_{2} \mathrm{O}_{3}(2 \mathrm{~nm}) /\right.$ $\left.\mathrm{Co}(5 \mathrm{~nm}) / \mathrm{Ir}_{20} \mathrm{Mn}_{80}(15 \mathrm{~nm}) / \mathrm{Au}\right)$ has been deposited in a commercial Alcatel sputtering system in a 4 mTorr argon plasma. The tunnel barrier is formed by postoxidation of a $1.5 \mathrm{~nm}$ thick aluminum layer in a $2 \mathrm{mTorr} \mathrm{Ar} / 2 \mathrm{mTorr} \mathrm{O}_{2} \mathrm{rf}$ plasma during $1 \mathrm{~min}$. For magnetotransport measurements, tunnel junctions of circular shape with dimensions ranging from 300 to $10 \mu \mathrm{m}$ are patterned in four steps using optical lithography and ion beam etching processes. ${ }^{10}$ The annealing process of the junction has been performed at $280^{\circ} \mathrm{C}$ during 15 min, under a 180 Oe saturating magnetic field applied perpendicularly to the steps. 


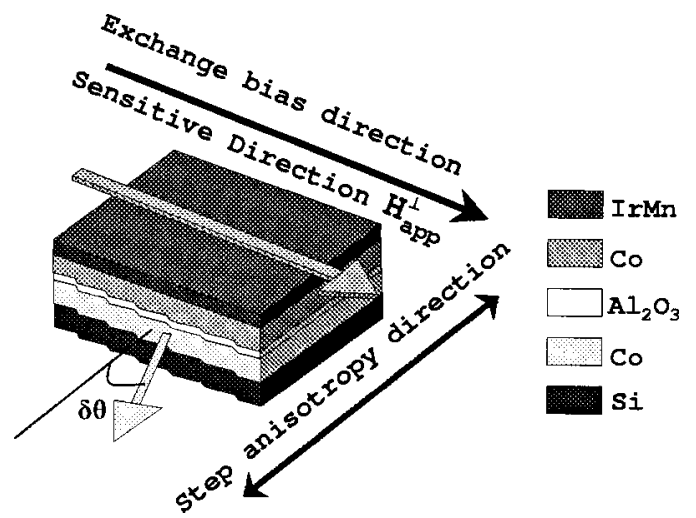

FIG. 1. Principle of low field measurements. The softer electrode magnetization is forced to rotate coherently around this equilibrium position by an angle $\delta \theta$. The hard magnet is pinned along the exchange bias direction perpendicular to steps.

In Fig. 2(a), we present the low-field behavior, at room temperature (RT) of the tunnel magnetoresistance of a 200 $\mu \mathrm{m}$ wide junction. The field $H_{\text {ap }}^{\perp}$ is oriented along the exchange bias direction corresponding to the hard magnetic axis of the softer magnet. We can observe that the resistance depends linearly on $H_{\mathrm{ap}}^{\perp}$. In addition, the signal plotted in Fig. 2(a), corresponding to three sweeps in field is seen to be accurately reversible. This originates from the coherent rotation mechanism experienced by the bottom Co magnetization away from its equilibrium position under the influence of $H_{\text {ap }}^{\perp}$
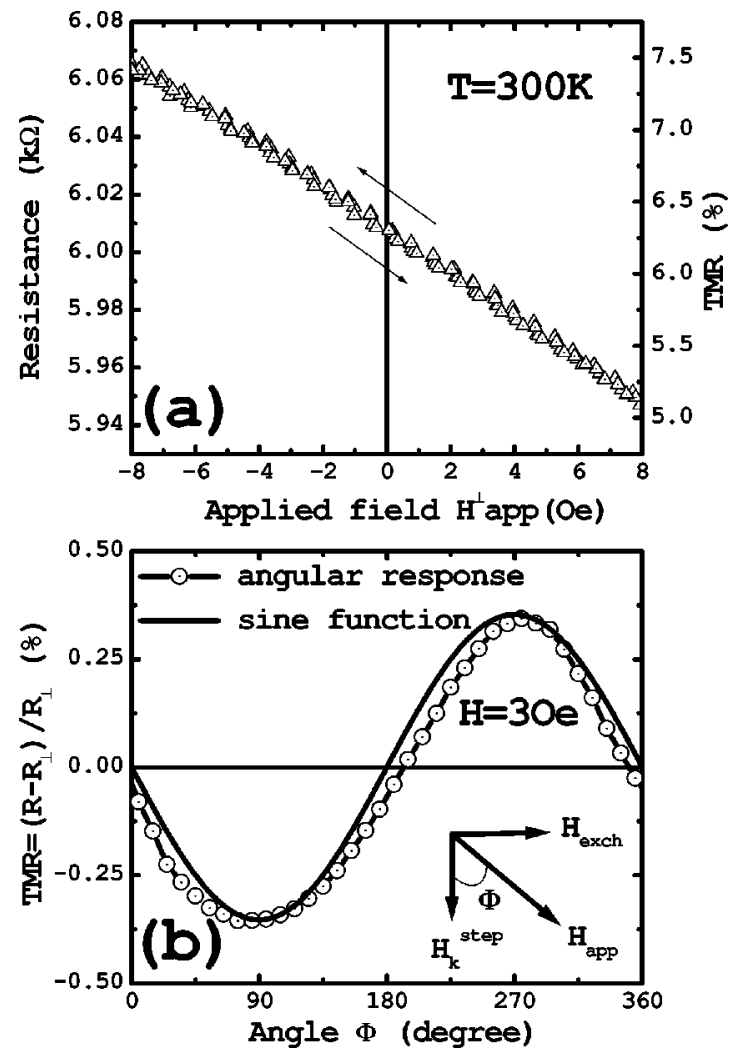

FIG. 2. (a) (- $\triangle$-) Low field behavior of the resistance at RT. $H_{\text {ap }}$ is aligned with the exchange bias direction. The signal was measured to be reversible by performing three minor loops. (b)(-๑-) Angular measurement of the TMR at RT. The straight line reproduces a sine curve. in this range $( \pm 8 \mathrm{Oe})$. In this regime, the slight angle variation $\delta \theta$ of the macrospin counted from the step direction (see Fig. 1) is responsible for the linear tunnel magnetoresistance depicted in Fig. 2(a). This angle variation leads to a relative sensitivity defined as $\delta R /\left(R_{\|} \times \delta H_{\text {ap }}\right)$ of about $0.12 \% \mathrm{Oe}^{-1}$. The expected sensitivity can simply be expressed as $\mathrm{TMR} /\left(2 H_{K}^{\text {step }}\right) \simeq 0.12 \% \mathrm{Oe}^{-1}$, where $\mathrm{TMR} \simeq 24 \%$ is the normalized difference of the resistance measured between the antiparallel and parallel magnetic configuration. Note that there is good agreement between the expected and measured sensitivity. In light of the expression of the sensitivity versus $H_{K}^{\text {step }}$, one must be aware that the sensitivity may be strongly improved by lowering $H_{K}^{\text {step }}$. This can be easily realized either by replacing the soft $\mathrm{Co}$ layer by a $\mathrm{Ni}_{80} \mathrm{Fe}_{20}$ layer or by depositing thicker films. ${ }^{11}$ However, previous studies have revealed the occurrence of quadratic effects and higher orders perturbations ${ }^{12}$ when $H_{K}^{\text {step }}$ approaches the dipolar coupling field and/or the applied field. These nonlinear effects strongly affect angular measurements. In other words, $H_{K}$ must be generally adapted to the desired function either field sensing (low anisotropy) or high precision angular detection (high anisotropy). In the present case, the equilibrium position is fixed by the strong local anisotropy field $H_{K}^{\text {step }}$ induced by the topological modulation which has been intentionally fixed at 100 Oe to avoid nonlinear effects. The study of the exchange bias properties of the top electrode by angular analysis, which is discussed in the following, partly explains our choice of a large $H_{K}^{\text {step }}$.

Indeed, angular measurements, i.e., measuring the resistance versus the orientation of a fixed applied field in the plane of the junction, constitutes a more powerful method to probe the linear property of the output tunnel signal versus $H_{\text {ap }}$. A perfect linear signal should then be described by a sine-like shape angular response corresponding to the field component $H_{\text {ap }}^{\perp}$ along the sensitive direction (Fig. 1). Inversely, in the low-field range, any distortion from the ideal sine-like variation has to be interpreted as the consequence of a nonideal behavior of the magnetic electrodes. ${ }^{12}$ Figure 2(b) presents the angular variation of the tunnel magnetoresistance versus the angle $\Phi$ between the applied field $\left(H_{\text {ap }}\right.$ $=3 \mathrm{Oe}$ ) and the step direction. The sensitive direction is here identified by $\Phi=90^{\circ}$. For clarity, the normalization of the resistance has been performed from the state $R_{\perp}$ corresponding to the perpendicular arrangement stabilized at zero field. This explains the respective positive and negative sign depending on the value of the angle $\Phi$. The signal has a reversible quasi sine-like shape, with a peak to peak magnitude of $0.7 \%$. The sensitivity approaches $0.12 \% O e^{-1}$ which corroborates the previous results. The minimum (maximum) of the resistance is obtained close to $\Phi=90^{\circ}\left(\Phi=270^{\circ}\right)$ i.e., when $H_{\text {ap }}$ is parallel (antiparallel) to the exchange bias direction leading to the minimization (maximization) of the relative angle between both electrodes.

However, the distortion from the sine shape, evidenced on Fig. 2(b), reveals a smaller resistance than expected. This reduction, originating from a reduction of the effective angle between the two magnetizations, may have two sources: the magnetization deviation of one or the other electrode. In a previous article, ${ }^{12}$ we have shown that the nonlinear response 


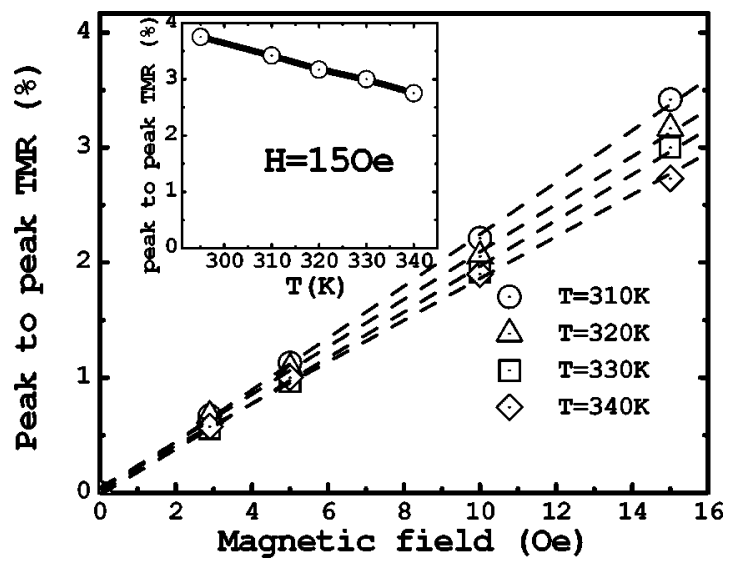

FIG. 3. Peak to peak magnitude of the angular response vs the applied field for different temperatures. The inset evidences the linear drop down of sensitivity when the temperature increases.

of the softer electrode must be visualized through: (1) a phase shift in the sine response and (2) a quadratic effect at a double frequency. This is clearly not the case here suggesting that the nonlinearity observed originates mainly from the hard electrode behavior. The angle reduction may result from a slight motion of the biased layer interacting with the external field as well as the softer layer through the orange peel coupling. ${ }^{13}$ The larger discrepancy is obtained for $\Phi=0^{\circ}$ and $\Phi=180^{\circ}$ corresponding to a perpendicular arrangement of the magnetizations, i.e., when the torque imposed by the soft layer on the biased one is maximal. The study of the angular response versus the temperature presented below will partly confirm this assumption.

In Fig. 3, we have reported the total amplitude of the angular response in the [0-15 Oe] field range for different temperatures $T$ ranging from 310 to $340 \mathrm{~K}$. The signal is again shown to be linear versus the applied field $H_{\text {ap }}$ with the slope of the curve (sensitivity) smoothly decreasing with $T$. For illustration, the thermal dependence of the sensitivity measured for $H_{\mathrm{ap}}=15 \mathrm{Oe}$, shown in the insert of Fig. 3, presents a linear relative variation of about $-27 \%$ between 295 and $340 \mathrm{~K}\left(600 \mathrm{ppm} \mathrm{K}^{-1}\right)$. This loss of sensitivity may be attributed to a more and more important motion of the hard layer around its nominal pinning position when the temperature increases. In order to investigate the role played by the exchange coupling between the AF material and the "top" electrode, junctions have been annealed with a field applied in a direction parallel to the vicinal steps to obtain a two parallel easy axes. This configuration allows us to observe clearly defined antiparallel states of the magnetizations at low temperature. In Fig. 4 we have plotted both the exchange field $H_{\text {exch }}$ and the TMR (extracted both from the TMR loops) as a function of temperature. We note that $H_{\text {exch }}$ decreases continuously while the temperature rises. On the other hand, two regimes can be distinguished in the TMR variation. The first one, for $T<250 \mathrm{~K}$, corresponds to a bias exchange field strong enough to pin the hard layer and form a large antiparallel plateau. The second regime, taking place above $T=250 \mathrm{~K}$, is associated with a significant drop of the TMR, reflecting a partial motion of the hard magnet. For temperatures larger than $250 \mathrm{~K}$, the exchange coupling be-

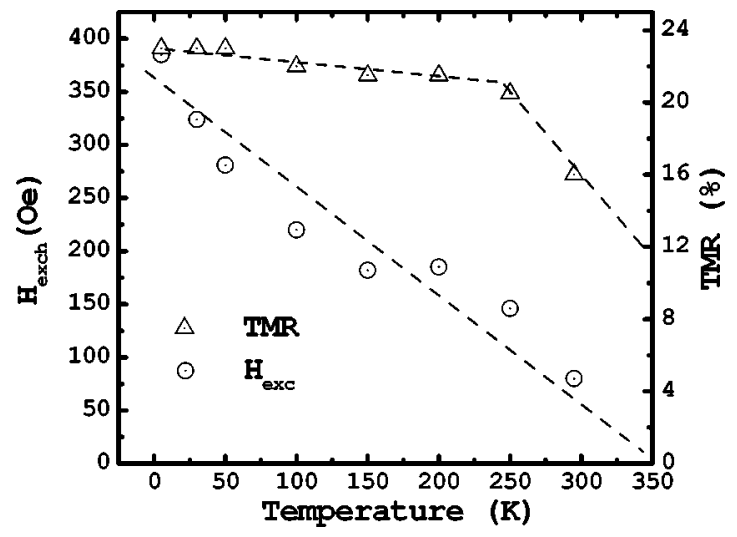

FIG. 4. $H_{\text {exch }}$ and TMR vs temperature. The lines are guides for the eyes.

tween the AF material (IrMn) and the "top" ferromagnetic electrode (Co) is not strong enough to allow an independent reversal of each electrode. Therefore both the distortion and the reduced sensitivity observed at room temperature can be qualitatively explained by a partial loss of exchange properties. As described in the literature, the exchange bias properties are known to be strongly dependent on the crystalline quality of the AF films. For example the grains size ${ }^{14}$ and/or the texture ${ }^{15}$ of the AF layer may influence the existence and the magnitude of the exchange field. In the "top" configuration, i.e., when the AF film is grown above a thin amorphous barrier (the present case), the growth conditions may alter the crystalline quality of the AF layer. Indeed, complementary $\mathrm{x}$-ray diffraction and high resolution transmission electron microscopy studies detailed elsewhere ${ }^{16}$ have revealed how a lack of the (111) texture of the IrMn film could give rise to a loss of the exchange bias property. In addition, it is known that a distribution of blocking temperatures can be responsible of the "pinned" layer motion during angular measurements at RT. ${ }^{17}$

In conclusion, we have reported an original method to realize a magnetic field sensor based on a spin dependent tunneling effect. By combining the use of step bunched substrates with exchange biasing of the top electrode, we succeeded in linearizing the signal supplied by magnetic tunnel junctions. The optimization of the sensor performances at high temperatures requires the improvement of the crystalline properties of the IrMn layer or the use of an alternate AF material.

This work has been partially supported by the European Commission through Grant Nos. BR CT98-0657 ("Tunnelsense") and E-32464 ("Massdots").

${ }^{1}$ J. S. Moodera, L. R. Kinder, T. M. Wong, and R. Meservey, Phys. Rev. Lett. 74, 3273 (1995).

${ }^{2}$ S. S. P. Parkin, K. P. Roche, M. G. Samant, P. M. Rice, R. B. Beyers, and R. E. Scheurlein, J. Appl. Phys. 85, 5828 (1999).

${ }^{3}$ M. Tondra, J. Daughton, D. Wang, R. S. Beech, A. Fink, and J. A. Taylor, J. Appl. Phys. 83, 6688 (1998).

${ }^{4}$ J. Daughton, J. Brown, E. Chen, R. Beech, A. Phom, and W. Kude, IEEE Trans. Magn. 30, 4608 (1994).

${ }^{5}$ F. B. Mancoff, J. H. Dunn, B. M. Clemens, and R. L. White, Appl. Phys. Lett. 77, 1879 (2000).

${ }^{6}$ M. Tondra, J. Daughton, C. Nordman, D. Wang, and J. Taylor, J. Appl. Phys. 87, 4679 (2000). 
${ }^{7}$ S. A. Wolf et al., Science 294, 1488 (2001).

${ }^{8}$ F. Montaigne, P. Gogol, J. Briatico, F. Nguyen Van Dau, F. Petroff, A. Fert, and A. Schuhl, Appl. Phys. Lett. 76, 3286 (2000).

${ }^{9}$ J. Nogués and I. K. Schuller, J. Magn. Magn. Mater. 192, 203 (1999).

${ }^{10}$ F. Montaigne, J. Nassar, A. Vaurès, F. Nguyen Van Dau, F. Petroff, A. Schuhl, and A. Fert, Appl. Phys. Lett. 73, 2829 (1998).

${ }^{11}$ A. Encinas, F. Nguyen Van Dau, A. Schuhl, F. Montaigne, M. Sussiau, and P. Galtier, J. Magn. Magn. Mater. 198-199, 15 (1999).
${ }^{12}$ H. Jaffrès, D. Lacour, F. Nguyen VanDau, J. Briatico, F. Petroff, and A. Vaurès, Phys. Rev. B 64, 064427 (2001).

${ }^{13}$ L. Néel, C. R. Acad. Sci. Paris 255, 1676 (1962).

${ }^{14}$ H. N. Fuke, K. Saito, M. Yoshikawa, H. Iwasaki, and M. Sahashi, Appl. Phys. Lett. 75, 3680 (1999).

${ }^{15}$ J. van Driel, F. R. de Boer, K.-M. H. Lenssen, and R. Coehorn, J. Appl. Phys. 88, 975 (2000).

${ }^{16}$ D. Lacour et al. (unpublished).

${ }^{17}$ J. P. Nozières, S. Jaren, Y. B. Zhang, A. Zeltser, K. Pentek, and V. S. Speriosu, J. Appl. Phys. 87, 3920 (2000). 